\title{
First Results of Experimental and Theoretical Investigations on Sextet States of Doubly-Excited Five-Electron Ions
}

\author{
J. H. Blanke and B. Fricke \\ Fachbereich Physik, Universität Kassel, D-3500 Kassel, Germany
}

and

P. H. Heckmann and E. Träbert

Experimentalphysik III, Ruhr-Universität Bochum, D-4630 Bochum 1, Germany

Received September 12, 1991; accepted December 3,1991

\begin{abstract}
In continuation of our previous work on doubly-excited ions with three and four electrons we present the first results on optical transitions in the term system of doubly-excited jons with five electrons. Transitions between such sextet states were identified in beam-foil spectra of the ions nitrogen, oxygen and fluorine. Assignments were first established by comparison with Multi-Configuration Dirac-Fock calculations. Later assignments were aided by Multi-Configuration Hartree-Fock calculations (see the contribution by G. Miecznik et al. in this issue). Decay curves were recorded for all six candidate lines. The lifetime results are compared to theoretical values which confirm most of the assignments qualitatively.
\end{abstract}

\section{Introduction}

Optical transitions between doubly-excited states of fewelectron ions have been studied extensively. An excellent. over-view is given by Mannervik [1]. So far, most of the information has been gained on the quartet states of threeelectron ions. Most of these observations have been using the beam-foil light source, in which these core-excited states are readily excited. This experimental method was also employed when investigating the quintet states of doublyexcited four-electron ions, the knowledge on which could recently be extended to $n=3$ states of several light ions [2-4].

In contrast to the doubly-excited quartet and quintet states, the sextet term system of doubly-excited five-electron ions shows only a single state with $n=2$, i.e. $1 s 2 s 2 p^{36} S^{\circ}$. Whereas for the quartet and quintet state problem the $n=2-n^{\prime}=2$ (intra-shell) transitions $1 s 2 s 2 p^{4} P^{0}-1 s 2 p^{24} P$ and $1 s 2 s 2 p^{25} P-1 s 2 p^{35} S^{\circ}$, respectively, were the first to be found and identified for nearly all light elements [1], there is no $n=2, \Delta n=0$ sextet transition. The first candidate for an identification of a sextet transition in a core-excited ion is an $n=2-n^{\prime}=3$ transition of the type $1 s 2 s 2 p^{36} P^{\circ}$ $1 s 2 s 2 p^{2} 3 l^{6} L$. The only other $n=3$ even state belongs to the configuration $1 s 2 p^{3} 3 p$. A level scheme for the case of $\mathrm{N}$ III is presented in Fig. 1.

The sextet states are stable against dipole-radiation decay to singly-excited five-electron states and against autoionization decay to singly excited four-electron states. In both cases the final states would possess a $1 s^{2}$ core and cannot couple to sextet states; thus the transitions would violate the $\Delta S=0$ selection rule. Furthermore, the sextet states in $\mathrm{N}$ III, $\mathrm{O}$ IV and F V, which are investigated here, lie below the $1 s 2 s 2 p^{25} P$ limit, so that this autoionization decay channel is also blocked. Mixing with core-excited quartet levels has not been included in our treatment, because it was considered to be of lesser importance. It has been shown previously (for example for C II in $[5,6]$ ) that sextet states are populated in the beam-foil source, by observing (via electron spectroscopy) the autoionization process of the $1 s 2 s 2 p^{36} S^{\circ}$ level. We are not aware of any previous observation of optical transitions between sextet levels of coreexcited ions. Theoretical wavelengths for such transitions have been calculated $[5,7]$ previously for some transitions in B I and C II.

In this paper we concentrate on the five-electron ions of $\mathrm{N}, \mathrm{O}$ and $\mathrm{F}$. Neither experimental nor theoretical data were available at the start. We obtained theoretical wavelength data by the Multi-Configuration Dirac-Fock method (MCDF) as employed in the latest version of the Desclaux code [8]. At present our calculation includes only the configurations of the complexes of the levels involved. This is a shortcoming, but our previous investigations $[3,4]$ have shown that already this approximation can provide meaningful data for a comparison of theoretical and experimental transition energies along the isoelectronic sequence; such an analysis is a most useful tool which aids the identification of transitions. During the course of our investigation theoretical results obtained by Brage and Miecznik [9] when using

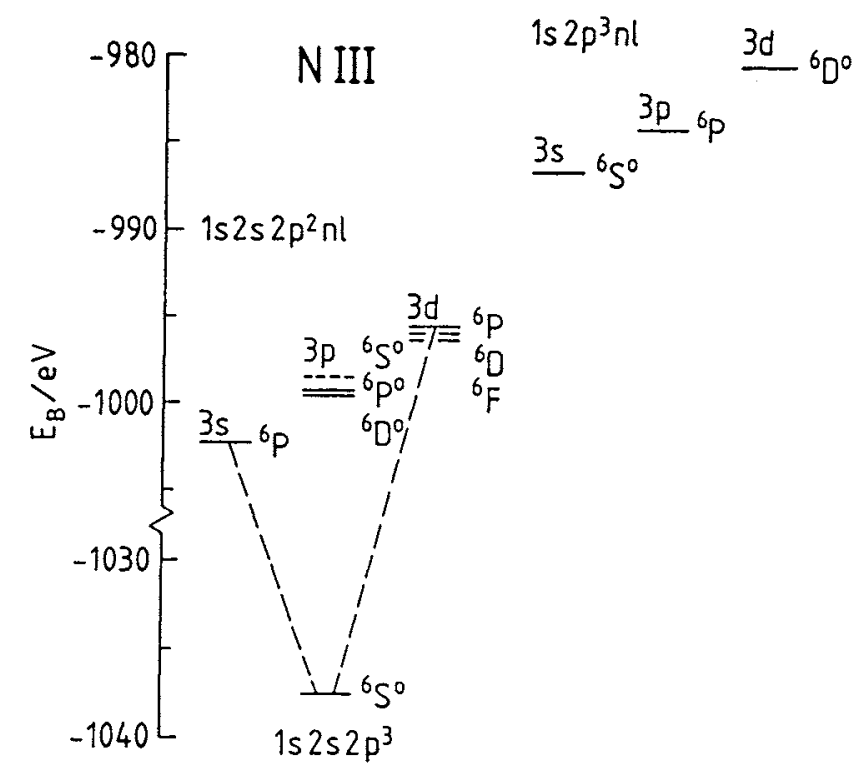

Fig. 1. Term diagram of doubly-excited sextet states for the case of $N$ III. Lines indicate transitions identified in the course of this work and discussed in the text. Near $E_{\mathrm{B}}=-982 \mathrm{eV}$ the $1 s 2 s 2 p^{2}{ }^{5} P$ limit is indicated 
an MCHF code became available; their data were most helpful for the continued search for the proper lines. Their results are also published in this issue.

Experimentally we relied on the beam-foil facility at Bochum which encompasses an EUV-spectrometer with a sufficiently high efficiency for the wavelength range in which the sextet transitions of the ions $\mathrm{N}, \mathrm{O}$ and $\mathrm{F}$ are expected. It should be noted, however, that these lines are weak (probably because their upper levels are not significantly repopulated by cascades, since those cascading states would easily autoionize). They are often blended and their mostly short lifetimes are at the limit of measurability, in particular considering the low ion beam energies required for optimum production.

After a brief discussion of our theoretical method and of the experimental facilities we discuss possible line identifications and decay curve evaluations of both transitions $1 s 2 s 2 p^{36} S^{\circ}-1 s 2 s 2 p^{2} 3 s^{6} P$ and $1 s 2 s 2 p^{36} S^{0}-1 s 2 s 2 p^{2} 3 d^{6} P$ for the elements $\mathrm{N}, \mathrm{O}$ and $\mathrm{F}$ in detail.

\section{Theory}

For our calculations the latest version of Desclaux' MultiConfiguration Dirac-Fock program [8] was used. The Dirac-Fock equations were solved separately for all sextet states which arise from the complexes of the odd $n=2$ configurations and the even $n=3$ configurations. These are $1 s 2 s 2 p^{3}$ and $\left(1 s 2 s 2 p^{2} 3 s, 1 s 2 s 2 p^{2} 3 d, 1 s 2 p^{3} 3 p\right)$, respectively. after evaluation of all possible levels for all values of $J$, the levels were grouped in certain $L S$-terms (averaged over the fine structure sublevels statistically in order to obtain $L S$-term energies), because the fine structure was not resolved in the spectra.

From our previous studies of doubly-excited three- and four-electron ions $[3,4,10]$ we learned that it is often sufficient to calculate upper and lower states within the manifold of the configurations of their complexes. The comparison of experimental and theoretical transition energies along the isoelectronic sequences even then shows simple trends. Their analysis is a powerful tool, provided the term diagram is fairly simple. For example, there must not be states of other Rydberg series which might lead to crossings with the states in question. In the case of the sextet states, such crossings do certainly not exist for the states $1 s 2 s 2 p^{36} S^{0}, 1 s 2 s 2 p^{2} 3 s^{6} \mathrm{P}$ and $1 s 2 s 2 p^{2} 3 d^{6} P$. On the other hand, the $1 s 2 p^{3} 3 p^{6} P$ state is difficult to calculate, because the mixing with members of the $1 s 2 s 2 p^{2} n l^{6} P$ Rydberg series has to be considered. This case, therefore, is outside the scope of our present investigation. For the remaining difference between experimental and theoretical transition energies one can deduce the relation

$E_{\text {Experiment }}-E_{\mathrm{MCDF} \text { in complex }}=a+b / Z+C / Z^{2}+\ldots$,

which is valid for the MCDF approach up to $Z=15$, where second order relativistic effects begin to become important (magnetic correlation). The leading term in eq. (1) is a constant. This observation was used, for example, in the investigations on the quintet transitions $1 s 2 s 2 p^{25} P-1 s 2 s 2 p 3 d^{5} P^{\circ}$, ${ }^{5} D^{\circ}[3,4]$. For almost neutral systems, however, the subsequent hyperbolic terms might become important. As an estimate of their contribution, Fig. 2 shows the example of the quartet transitions $1 s 2 s 2 p^{4} P^{\circ}-1 s 2 s 3 s^{4} S$ and $1 s 2 s 2 p^{4} P^{\circ}-$ $1 s 2 s 3 d^{4} D$ which are somehow related to the sextet transitions which we consider below. The hyperbolic terms in

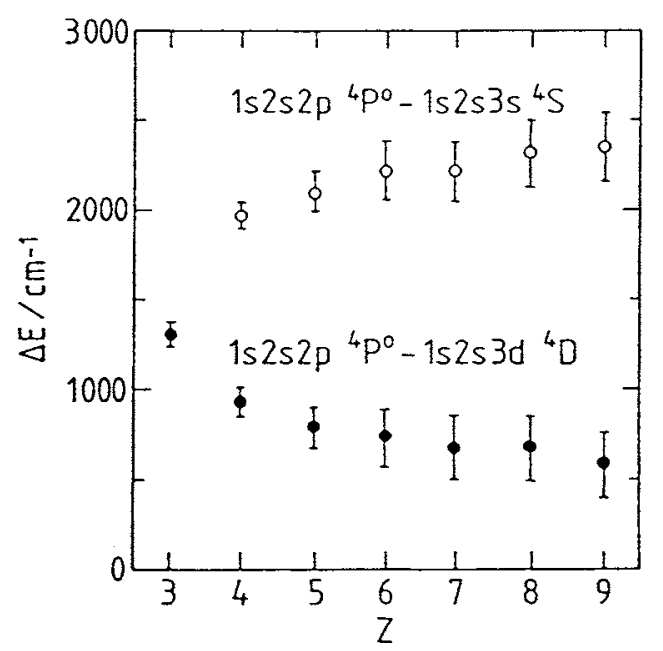

Fig. 2. Deviations between experimental and theoretical transition energies for the transitions $1 s 2 s 2 p^{4} P^{\circ}-1 s 2 s 3 s^{4} S$ and $1 s 2 s 2 p^{4} P^{\circ}-1 s 2 s 3 d^{4} D$ in threeelectron ions. Experimental data for $\mathrm{Li}, \mathrm{Be}$ and $\mathrm{B}$ are taken from [1], and for $\mathrm{C}, \mathrm{N}, \mathrm{O}$ and $\mathrm{F}$ from [14]. Theoretical values are calculated with an MCDF code, in a "complex" approach explained in the text

eq. (1) are evident for the systems Li I-B III (Fig. 2). A similar effect may be expected to be present and important for the sextet transitions in N III.

The sextet states couple to maximum spin quantum number $S=5 / 2$, and no different parents are involved in the coupling. Following the rules of Wood and Pyper [12], we therefore do not expect that any extra energies like those described by Huang [13] contribute to our case. Nevertheless we ascertained that in a non-relativistic MCDF calculation ( $c=10^{10}$ a.u.) all $J$-sublevels of a given $L S$ term have the same total non-relativistic energies. The total energy of these self-consistent calculations also includes contributions of the Breit interaction as a perturbation (magnetic interaction and retardation). Contributions of the self-energy are taken into account by using the effective nuclear charge $Z$, which is calculated from an analogue hydrogenic orbital with the same expectation value for $r$ as the MCDF orbital in question. The Uehling potential is used for the vacuum polarization. The existing MCDF code was extended in order to be able to calculate transition probabilities, too. For this purpose, upper and lower states of the transition are calculated independently. The consequent nonorthogonality of the orbitals is corrected in part by multiplying the transition matrix elements by the overlaps of the non-active orbitals. From our previous work on quintets and quartets $[3,4]$ we expect that such calculations which incorporate mixing within the complex, will give reliable and meaningful results for the sextet states considered.

\section{Experiment}

The experiments employed the beam-foil method and were done at the $4 \mathrm{MV}$ Dynamitron Tandem Accelerator Laboratory at Bochum. For all three elements studied ion beam currents of several $\mu \mathrm{A}$ were available. The ions were excited by being passed through a thin carbon foil (about 10-20 $\mu \mathrm{g} /$ $\mathrm{cm}^{2}$ ) which had been produced in a gas discharge. The foils typically lasted for five hours in a beam of $1 \mathrm{MeV}$ oxygen ions at a current of $2 \mu \mathrm{A}$ particles. Spectra were recorded using a $2.2 \mathrm{~m}$ grazing-incidence monochromator (McPherson Mod. 247) which viewed the ion beam at right angles. A ruled diffraction grating with $600 \mathrm{l} / \mathrm{mm}$ and a 
holographic one with $36001 / \mathrm{mm}$ were alternatively available. The high spectral resolution attainable with the latter was essential for the identification of some of the transitions, especially for F V $1 s 2 s 2 p^{3}{ }^{6} S^{\circ}-1 s 2 s 2 p 3 s^{6} P$. The linearity of the monochromator exit slit displacement was monitored by a $60 \mathrm{~mm}$ travel Heidenhain moiré fringe length gauge. A low-noise channeltron served as the detector. A detailed description of the apparatus can be found elsewhere [14].

For the purpose of lifetime measurements, intensity decay curves were recorded at the positions of all candidate lines of the sextet transitions in question (as well as for several possible alternative assignments). This was achieved by moving the carbon foil along the ion beam axis, the displacement being monitored by another $60 \mathrm{~mm}$ travel Heidenhain moire fringe length gauge. The signal at each foil position was normalized to a pre-set value of integrated ion beam current, as was done at each exit slit position in the recording of wavelength spectra.

The recorded decay curves are convolutions of the exponential decays (due to the lifetime of the upper levels) and the field-of-view of the detection system which is due to the finite spatial resolution of the apparatus. The upper level is usually repopulated by cascading transitions. In the evaluation procedure, two or three exponential functions and an assumed detection-window shape were fitted to each experimental decay curve. Starting values for the detectionwindow parameters of the fit procedure were obtained from geometrical considerations [15]. The final results for these parameters as obtained from the fits to the data were considered acceptable, if they deviated by less than 10 percent from the geometrical values.

The main decay component is then identified with the lifetime of the upper level, the others are assumed to relate to cascades or to blending transitions. For the $1 s 2 s 2 p^{2} 3 s^{6} P$ states cascading amounts to less than 2 percent of the amplitude of the main component. For the $1 s 2 s 2 p^{2} 3 d^{6} \mathrm{P}$ states there is expected a relatively strong cascade along the yrast line; its decay time is expected to exceed the lifetime of interest by at least one order of magnitude. Therefore, our fitting procedure should be applicable for these levels. More complicated are problems of blending which arise for some of the sextet transitions. This will be discussed in detail in Section 4.

\section{Results}

In a number of cases, spectra of those wavelength regions in which the sextet lines are expected were available from our previous measurements, although generally they had been recorded at higher beam energies than suitable for the population of sextet levels. Even though the decays of the sextet levels were not detectable in those spectra, they yielded information on intensities and line shapes of nearby lines originating from singly excited ions of higher charge states and were helpful for calibration and reference purposes.

For the present study we obtained spectra at least two different ion beam energies for each element, and of each of the regions where the sextet transitions were to be expected. We begin the discussion with the $1 s 2 s 2 p^{36} S^{\circ}-1 s 2 s 2 p^{2} 3 s^{6} P$ transition for N III, O IV and F V, to be followed by the $1 s 2 s 2 p^{36} S^{\circ}-1 s 2 s 2 p^{2} 3 d^{6} P$ transitions. Spectra are shown in

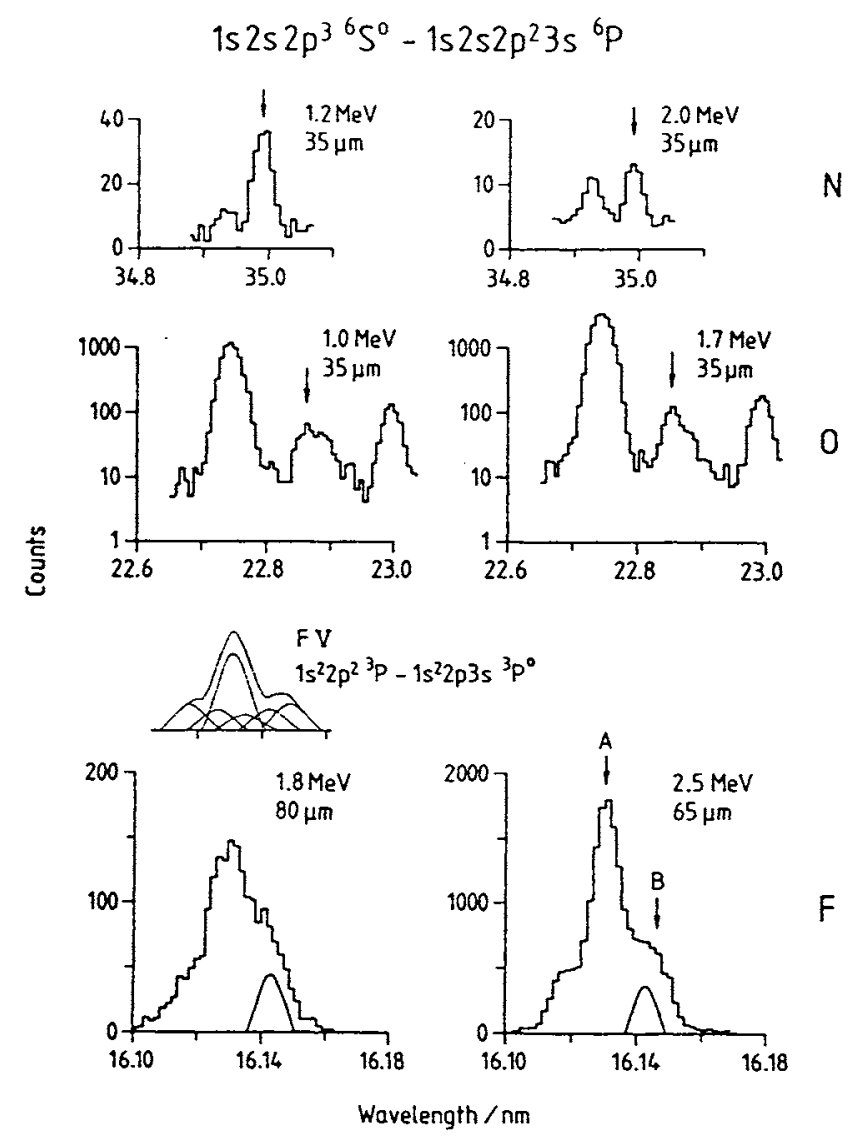

Fig. 3. The $1 s 2 s 2 p^{36} s^{\circ}-1 s 2 s 2 p^{2} 3 s^{6} P$ transition in beam-foil spectra of the elements $N, O$ and $F$, recorded at two different beam energies for each element. Beam energy and spectrometer slit width are indicated. The insert depicts the expected structure of the multiplet F V $1 s^{2} 2 p^{2}{ }^{3} P-1 s^{2} 2 p 3 s^{3} P^{0}$. An additional component (as sketched), which we ascribe to the transition of interest, is evidently present in the data. Decay curves recorded at positions $A$ and $B$ indicated in the bottom right spectrum are shown in Fig 6

Figs 3 and 4 . The lifetime analysis is discussed alongside the wavelength determination. Decay curves are presented in Figs 5 and 6 . The wavelength results are summarized in Table I, the lifetime data in Table II.

\subsection{The $1 s 2 s 2 p^{3}{ }^{6} S^{0}-1 s 2 s 2 p^{3} 3 s^{6} P$ transition}

For nitrogen two spectra were taken at beam energies of 1.2 and $2 \mathrm{MeV}$, respectively (Fig. 3). Two hitherto unidentified lines were located at the expected position of the N III $1 s 2 s 2 p^{36} s^{\circ}-1 s 2 s 2 p^{2} 3 s^{6} P$ transition. Relative intensities of both lines at the two beam energies suggest that the line at $\lambda=(34.922 \pm 0.007) \mathrm{nm}$ rather arises from an N IV tran-

Table I. Wavelengths of sextet transitions. The results of our beam-foil experiments are compared to data obtained by $M C D F$ and $M C H F$ calculations

\begin{tabular}{|c|c|c|c|c|}
\hline \multirow[b]{2}{*}{ Element } & \multirow[b]{2}{*}{$\begin{array}{l}\text { Experiment } \\
\lambda / \mathrm{nm}\end{array}$} & \multicolumn{2}{|c|}{ MCDF in complex } & \multirow[b]{2}{*}{$\begin{array}{l}\text { MCHF [9] } \\
\lambda / \mathrm{nm}\end{array}$} \\
\hline & & $\lambda / \mathrm{nm}$ & $\begin{array}{l}\text { Deviation } \\
\Delta E / \mathrm{cm}^{-1}\end{array}$ & \\
\hline \multicolumn{5}{|c|}{$1 s 2 s 2 p^{36} S^{\circ}-1 s 2 s 2 p^{2} 3 s^{6} P$} \\
\hline $\mathbf{N}$ & $34.983 \pm 0.005$ & 35.315 & $2687 \pm 50$ & 35.005 \\
\hline $\mathrm{O}$ & $22.863 \pm 0.004$ & 23.000 & $2605 \pm 80$ & 22.870 \\
\hline $\mathbf{F}$ & $16.139 \pm 0.003$ & 16.210 & $2714 \pm 120$ & 16.146 \\
\hline \multicolumn{5}{|c|}{$1 s 2 s 2 p^{36} S^{0}-1 s 2 s 2 p^{2} 3 d^{6} P$} \\
\hline $\mathbf{N}$ & $29.413 \pm 0.005$ & 29.649 & $2706 \pm 60$ & 29.425 \\
\hline $\mathrm{O}$ & $19.611 \pm 0.005$ & 19.700 & $2304 \pm 130$ & 19.620 \\
\hline $\mathbf{F}$ & $14.078 \pm 0.003$ & 14.121 & $2163 \pm 150$ & 14.081 \\
\hline
\end{tabular}




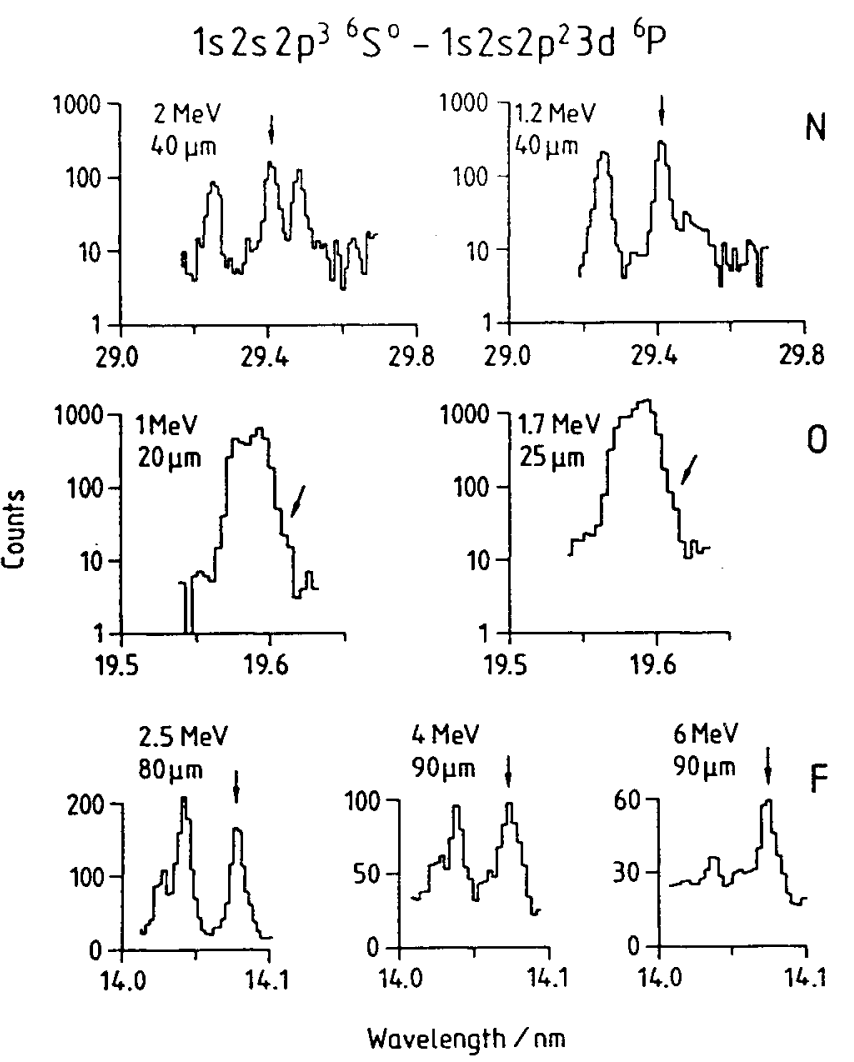

Fig. 4. The $1 s 2 s 2 p^{3}{ }^{6} S^{0}-1 s 2 s 2 p^{2} 3 d^{6} P$ transition in beam-foil spectra of the elements $N, O$, and $F$, recorded at two to three different beam energies each. The spectrometer slit widths are given for each recording

sition and that the line at $\lambda=34.983 \pm 0.005$ ) nm should be the sextet transition in question. However, the measured decay curve on this second line gave a value of $\tau=(103 \pm 15) \mathrm{ps}$ in gross disagreement with the calculated values (Table II). A second component in this decay curve, with an amplitude ten times smaller than that of the main component, features an apparent decay time of $\tau=(450 \pm 50) \mathrm{ps}$, which seems too long to associate it with the decay of interest. The cause of this lifetime discrepancy remains unclear, for a spectral line which on spectroscopic grounds is the most likely candidate for the wanted sextet transition.

For oxygen the situation is similar and not satisfying, either. Two spectra obtained at 1.0 and $1.7 \mathrm{MeV}$ beam energy are shown in Fig. 3. Again there are two possible candidates, at $\lambda=(22.863 \pm 0.004) \mathrm{nm}$ and at

Table II. Lifetimes of doubly-excited sextet states. Decay times extracted from beam-foil measurements are confronted with $M C D F$ and $M C H F$ calculational results

\begin{tabular}{llll}
\hline Element & $\begin{array}{l}\text { Experiment } \\
\tau / \mathrm{ps}\end{array}$ & $\begin{array}{l}\text { Theory } \\
\mathrm{MCDF}^{\mathrm{a}} \\
\tau / \mathrm{ps}\end{array}$ & $\begin{array}{l}\mathrm{MCHF}^{\mathrm{b}} \\
\tau / \mathrm{ps}\end{array}$ \\
\hline $1 s 2 s 2 p^{2} 3 s^{6} \mathrm{P}$ & & & \\
$\mathrm{N}$ & $103 \pm 15$ & 293 & 327.7 \\
$\mathrm{O}$ & $99 \pm 12$ & 125 & 140 \\
$\mathrm{~F}$ & $73 \pm 15$ & 64 & 71.22 \\
$1 s 2 s 2 p^{2} 3 d^{6} P$ & & & \\
$\mathrm{~N}$ & $66 \pm 12$ & 41 & 42.80 \\
$\mathrm{O}$ & $12 \pm 3$ & 15.6 & 14.9 \\
$\mathrm{~F}$ & $11 \pm 4$ & 6.7 & 6.689 \\
\hline
\end{tabular}

- Partial lifetimes, due to radiative transitions to $1 s 2 s 2 p^{36} P^{\circ}$ only

${ }^{b}$ Elaborate calculations explained in detail in Ref. [9]
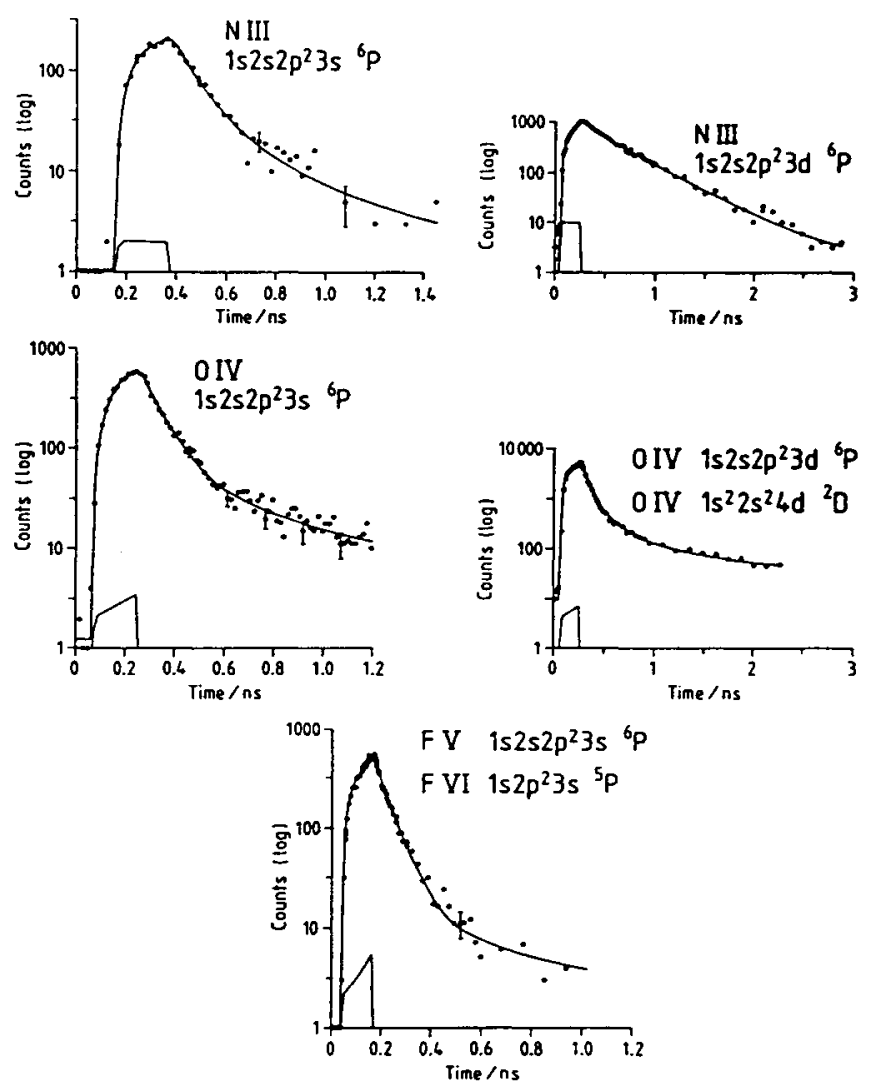

Fig. 5. Decay curves measured at five positions of sextet candidate lines $1 s 2 s 2 p^{3}{ }^{6} S^{0}-1 s 2 s 2 p^{2} 3 s^{6} P$ and $1 s 2 s 2 p^{36} S^{\circ}-1 s 2 s 2 p^{2} 3 d^{6} P$ for the elements $\mathrm{N}$, $O$ and $F$. A decay curve recorded at the presumed position of the $F \mathrm{~V}$ $1 s 2 s 2 p^{3}{ }^{6} S^{0}-1 s 2 s 2 p^{2} 3 s^{6} P$ transition is shown in Fig. 6

$\lambda=(22.890 \pm 0.003) \mathrm{nm}$. Although relative intensities in the limit of the error bars suggest that the first line is more likely due to a four-electron transition, it is tentatively assigned to the $\mathrm{O}$ IV $1 s 2 s 2 p^{36} S^{\circ}-1 s 2 s 2 p^{2} 3 s^{6} \mathrm{P}$ transition. At both positions decay curves were recorded and yielded lifetimes of $\tau=(95 \pm 12) \mathrm{ps}$ for the position at $\lambda=22.863 \mathrm{~nm}$ and $\tau=(370 \pm 50) \mathrm{ps}$ for the position at $\lambda=22.890 \mathrm{~nm}$. At this second position the $1 s^{2} 2 s^{2} 2 p^{2}{ }^{3} P-1 s^{2} 2 s 2 p^{2} 4 p^{3} S^{\circ}$ transition is known from the literature [16] and might be the

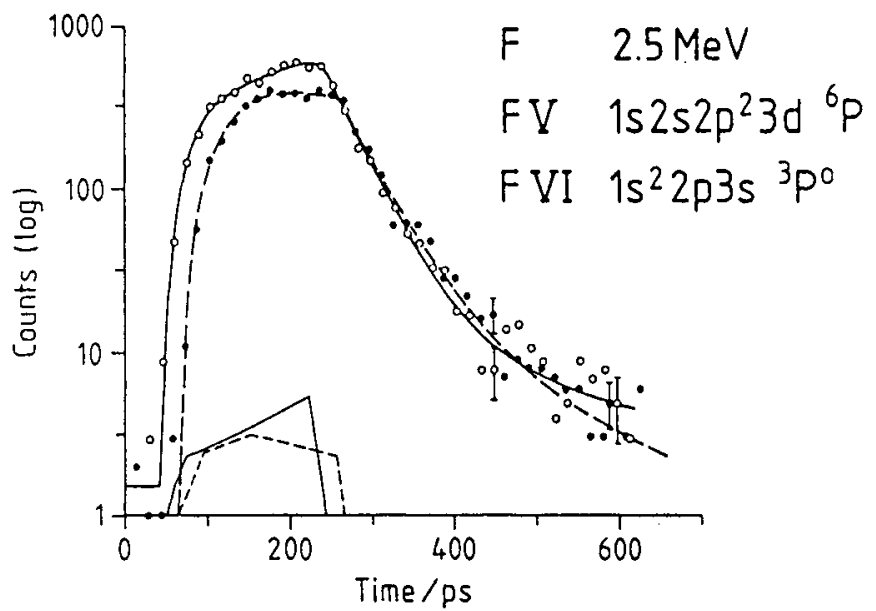

Fig. 6. Decay curves recorded at two positions $A(\lambda=16.132 \mathrm{~nm}$, full curve) and $B(\lambda=16.140 \mathrm{~nm}$, dashed curve) of the spectrum presented in Fig. 3. The detection efficiency curves as obtained from a fit to the data are indicated underneath. The two sets of curves have been drawn slightly displaced along the time axis. The analysis of the curve recorded at position A yields a single exponential decay component which is in agreement with the MCDF lifetime expected for $\mathrm{F}$ VI $1 s^{2} 2 p^{2}{ }^{3} P-1 s^{2} 2 p 3 s^{3} P^{\circ}$. At position B there is an additional contribution which we ascribe to the sextet transition F V $1 s 2 s 2 p^{3}{ }^{6} S^{\circ}-1 s 2 s 2 p^{2} 3 s^{6} P$ (Table II) 
Table III. Tentative results on the $1 s 2 s 2 p^{36} S^{\circ}-1 s 2 p^{3} 3 p^{6} P$ transition

\begin{tabular}{lllll} 
Element & $\begin{array}{l}\text { MCDF in complex } \\
\lambda / \mathrm{nm}\end{array}$ & $\begin{array}{l}\text { Expectation } \\
\lambda / \mathrm{nm}\end{array}$ & $\begin{array}{l}\text { Experiment tentative } \\
\lambda / \mathrm{nm}\end{array}$ & $\begin{array}{l}1 s 2 s 2 p^{2}{ }^{5} P-1 s 2 s 2 p 3 d^{5} P^{\circ}[3] \\
\lambda / \mathrm{nm}\end{array}$ \\
\hline $\mathrm{N}$ & 23.141 & $23.008 \pm 0.027$ & & $23.075 \pm 0.003$ \\
$\mathrm{O}$ & 16.252 & $16.186 \pm 0.013$ & $16.198 \pm 0.005$ & $16.227 \pm 0.002$ \\
$\mathrm{~F}$ & 12.065 & $12.029 \pm 0.008$ & & $12.058 \pm 0.002$ \\
\hline
\end{tabular}

"Expectation" is derived from the isoelectronic trend indicated by the calculation, assuming a missing correlational part of the total energy of the lower state of approximately $2500 \pm 500 \mathrm{~cm}^{-1}$

origin of this line. Hence we are left with the line at $\lambda=22.863 \mathrm{~nm}$. The wavelength is in good agreement with the predictions for a sextet transition (Table I), but the lifetime is not. For both cases, N III and O IV $1 s 2 s 2 p^{36} S^{o_{-}}$ $1 s 2 s 2 p^{2} 3 s^{6} P$, the measured decay curves are dominated by the main component as can be seen in the logarithmic plot of Fig. 5. Furthermore the decay times are long enough not to be affected by a possibly incorrectly fitted detection window function. Therefore the lifetime extraction from the measured curves does not seem problematic.

For fluorine the situation turned out to be more satisfying, although the F V $1 s^{2} 2 p^{2}{ }^{3} P-1 s^{2} 2 p 3 s^{3} P^{o}$ transition in the singly-excited ion is definitely present, too, at the expected position of the sextet line, with a wavelength value (statistically averaged over the fine structure) of $\lambda=16.1322 \mathrm{~nm}$. In the following we want to demonstrate that the sextet line is hidden within the profile of this line. Fortunately, for this problem the diffraction grating with the higher groove density of $3600 \mathrm{l} / \mathrm{mm}$ could be used. (Mechanical reasons allow us to access a wavelength region from 5 to $20 \mathrm{~nm}$ when using this grating.) Spectra were taken at ion energies of 1.8 and $2.5 \mathrm{MeV}$. For the spectrum at $1.8 \mathrm{MeV}$, wider slits of $80 \mu \mathrm{m}$ (instead of $65 \mu \mathrm{m}$ ) were used to counter the lower signal. The fitting procedure allowed for nine components to simulate the full line cluster. Six components represented the transition $\mathrm{FV} \quad 1 s^{2} 2 p^{2}{ }^{3} \mathrm{P}$ $1 s^{2} 2 p 3 s^{3} P^{o}$ with known relative line positions [16] and relative intensities [17]. The relative positions of the three components of the sextet transitions were taken from the calculated fine structure of the upper ${ }^{6} P$ state, which is one order of magnitude smaller than the fine structure of the ${ }^{3} P$ levels. For the upper level of both transitions statistical populations (like generally observed in these studies) were assumed and thus relative intensities inside each multiplet could be predicted. For both cases the actual calculations do not show any irregularities for the transition matrix elements between specific $J$-sublevels. Because of the predictable relative intensities and fine structure intervals, the fitting problem is reduced to two relative positions (of the multiplets) and two relative intensities.

In Fig. 3 the calculated line shape of the ${ }^{3} P-{ }^{3} P^{\circ}$ transition alone is indicated above the appropriate spectrum recorded at $2.5 \mathrm{MeV}$ beam energy (taking the spectral resolution function into account). The broad line shows the position and line shape of the fitted sextet transition in both cases. Within the error bars, its position is similar in both measurements. The different relative intensities, when comparing to the $\mathrm{F} V$ triplet line complex, would indicate an origin of the hidden line as from a lower charge state than that of the $F \mathrm{~V}$ transition. It seems reasonable to assume that the excitation function of a core-excited ion of charge $q+$ is somewhat different from the excitation functions of singly excited ions $[18,19]$, and this would fit in with our findings. At an ion-beam energy of $2.5 \mathrm{MeV}$, two lifetime measurements were performed at positions A and B (Fig. 3). At position $A$ the analysis gave a single component of $\tau=(42 \pm 3) \mathrm{ps}$ in good agreement with the calculated value of $40.4 \mathrm{ps}$ for the F V $1 s^{2} 2 p 3 s^{3} P^{\circ}$ level. At position $\mathrm{B}$ there is a second component detectable in the decay slope (Fig. 6). The experimental value of $\tau=(73 \pm 15) \mathrm{ps}$ is in agreement with the results of both theoretical approaches for the upper sextet state (Table II).

\subsection{The $1 s 2 s 2 p^{3}{ }^{6} S^{0}-1 s 2 s 2 p^{3} 3 d^{6} P$ transition}

Starting again with nitrogen, we found a line at $\lambda=(29.413 \pm 0.005) \mathrm{nm}$ with a dependence of the relative line intensity on the ion energy $(1.2$ and $2.0 \mathrm{MeV})$ as expected for $\mathrm{N}^{2+}$ (Fig. 4). The relatively high intensity hints at this spectral feature might consist of more than just the sextet transition. The decay curve analysis confirmed this suspicion. There are two equally strong decay components with $\tau=(64 \pm 12) \mathrm{ps}$ and $\tau=(430 \pm 25) \mathrm{ps,}$, respectively. The first component is seen as an indication for a sextet component contributing to the line. The origin of the other component is not known.

In the case of oxygen there is again a problem of blending. The possible sextet candidate is found on the longwavelength slope of the line which originates from the known transition $\mathrm{O}$ IV $1 s^{2} 2 s^{2} 2 p^{2} P^{\circ}-1 s^{2} 2 s^{2} 4 d^{2} D$ which has two spectroscopically separable components at $\lambda=19.5863 \mathrm{~nm}$ and $\lambda=19.6009 \mathrm{~nm}$ [16]. A measurement with the higher-resolution grating ( 3600 lines $/ \mathrm{mm}$ ) and a slit width of $45 \mu \mathrm{m}$ resulted in a wavelength result of $\lambda=(19.611 \pm 0.005) \mathrm{nm}$ for the new line. (The highresolution data, recorded at only one beam energy, have not been included in Fig. 4, in favour of data recorded at lower resolution, but at two energies.) From the fine structure splitting of only $4.4 \mathrm{~cm}^{-1}$ of the upper ${ }^{2} D$ state one can exclude that the observed line is due to O IV $1 s^{2} 2 s^{2} 2 p^{2} P_{3 / 2}^{\circ}-1 s 2 s^{2} 4 d^{2} D_{3 / 2}$. A lifetime measurement at this position exhibits a weak component with $\tau=(12 \pm 3)$ ps besides the dominant component of $\tau=(73 \pm 5)$ ps. The stronger component is in good agreement with the MCDFlifetime result of $69.8 \mathrm{ps}$ for O IV $4 d^{2} D$.

In the spectrum of foil-excited fluorine there is a line at $\lambda=14.078 \mathrm{~nm}$. This line could be found in spectra taken at four different beam energies $(2.5,4,5$ and $6 \mathrm{MeV})$. From its relative intensities we conclude that an identification with both the quintet transition $1 s 2 p^{35} S^{\circ}-1 s 2 p^{2} 3 s^{5} P$ [4] and the sextet transition $1 s 2 s 2 p^{36} S^{\circ}-1 s 2 s 2 p^{2} 3 d^{6} P$ would seem justified. In Fig. 4 we show the spectra recorded at $2.5,4$, and $6 \mathrm{MeV}$. For comparison, the spectra include the nearby $\mathrm{F} \mathrm{V}$ $1 s^{2} 2 s^{2} 2 p^{2} P^{\circ}-1 s^{2} 2 s^{2} 4 s^{2} S$ transition with its two components. The decay curve (recorded at $5 \mathrm{MeV}$ ) shows two 
equally strong components with $\tau=(11 \pm 4) \mathrm{ps}$ and $\tau=(68 \pm 7) \mathrm{ps}$, respectively. MCDF calculations give a value of 55 ps for the quintet level [4], MCDF and MCHF calculations [9] predict $\tau=6.7 \mathrm{ps}$ for the sextet level of interest. Since some cascading might be present, this seems a satisfying result under these circumstances.

\subsection{The $1 s 2 s 2 p^{36} S^{0}-1 s 2 p^{3} 3 p^{6} P$ transition}

Finally we would like to comment on the third possible $n=2-n^{\prime}=3$ sextet transition, that is $1 s 2 s 2 p^{36} S^{0_{-}}$ $1 s 2 p^{3} 3 p^{6} P$. From the term diagram (Fig. 1) it is obvious that the $1 s 2 p^{3} 3 p^{6} P$ state is located among the two Rydberg level series $1 s 2 s 2 p^{2} n s, n d^{6} P$. Thus there might occur short range interactions, differently for each element, which can lead to considerable energy shifts or even irregularities in the transition probabilities. A reliable theoretical description of this complex situation is outside the scope of our MCDF calculations, but as a rough estimate we list (in Table III) the results for the ions $\mathrm{N}, \mathrm{O}$ and $\mathrm{F}$ for calculations inside the complex. Assuming that the main part of the deviation between experimental and theoretical transition energies for the two transitions $1 s 2 s 2 p^{36} S^{\circ}-1 s 2 s 2 p^{2} 3 s, 3 d^{6} \mathrm{P}$ is due to the missing correlation part of the lower state $1 s 2 s 2 p^{36} S^{\circ}$ (of approximately $2500 \pm 500 \mathrm{~cm}^{-1}$ ), one might correct the MCDF result by this contribution and obtain an improved expectation value for $1 s 2 s 2 p^{3}{ }^{6} S^{\circ}-1 s 2 p^{3} 3 p^{6} P$. This procedure, however, does not account for the additional shortrange interactions of the upper $n=3$ states. We recorded spectra in the appropriate wavelength regions, but they do not show any convincing candidate. There is but one faint line at $\lambda=(16.198 \pm 0.005) \mathrm{nm}$ which might be a candidate for a tentative assignment in O IV. For all three elements, however, there might be blendings with the quintet transition $1 s 2 s 2 p^{25} P-1 s 2 s 2 p 3 d^{5} P^{\circ}$ [3] (also listed in Table III). Thus this problem remains open.

\section{Discussion and conclusion}

Following the above analysis we first compare the stated experimental wavelengths with those from the MCDF approach (Table I and Fig. 7). Assuming a possible asymptotic slope for N III (see Section 2), the deviation is constant, as expected, for both transitions. The comparison

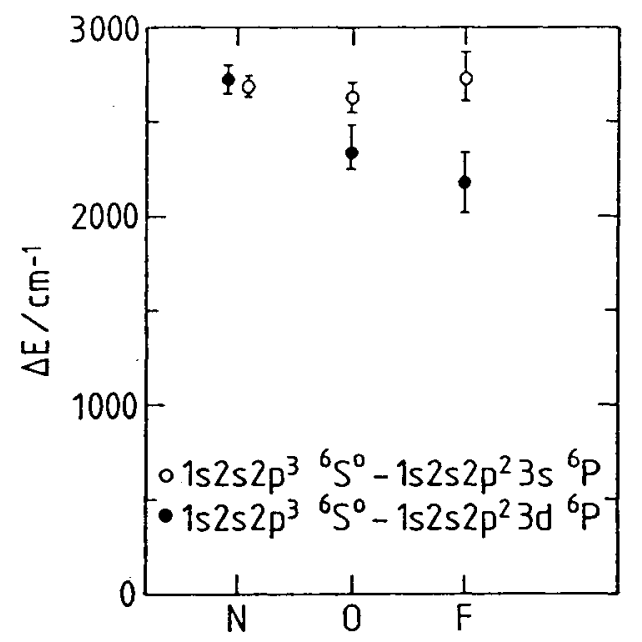

Fig. 7. Deviations between experimental and theoretical transition energies for the transitions $1 s 2 s 2 p^{36} S^{\circ}-1 s 2 s 2 p^{2} 3 s^{6} P$ and $1 s 2 s 2 p^{36} S^{\circ}-1 s 2 s 2 p^{2} 3 d^{6} P$. Theoretical values are calculated with the MCDF code, in a "complex" approach explained in the text with MCHF results is even more convincing and is discussed in detail in Ref. 9. The consistency of this comparison for all six transitions quoted is certainly a strong argument for this first identification of optical transitions between sextet states of doubly-excited five-electron ions.

Considering the lifetimes, however the results are not yet consistent. For the transition $1 s 2 s 2 p^{36} s^{\circ}-1 s 2 s 2 p^{2} 3 d^{6} P$ the experimental data confirm that short-lived components are embedded in the decay curves. At present blendings prevent quantitatively better results in all cases. The situation for $1 s 2 s 2 p^{3} S^{0}-1 s 2 s 2 p^{2} 3 s^{6} P$ is even more complex. For N III and $O$ IV there appears to be a mismatch with theory, while the data for F V look better. Miecznik and Brage, for example, discuss the possibility of further decay channels in detail [9]. The $1 s 2 s 2 p^{3}{ }^{6} S^{0}-1 s 2 p^{3} 3 p^{6} P$ transition remains a challenge for both theory and experiment.

\section{Acknowledgements}

We would like to thank T. Brage and G. Miecznik for joining us in our efforts concerning this problem. By enlightening discussions and by their reliable theoretical values they have assisted with the proper identifications of the sextet transitions. We thank the staff of the Bochum accelerator for running the machine so successfully. The excellent exciter foils were prepared by H. H. Bukow, while J. Doerfert, J. Granzow and C. Wagner helped with the experiment. The data acquisition system was granted by the Deutsche Forschungsgemeinschaft. Finally we would like to express our thanks to J. P. Desclaux for his continuous support. One of us (J.H.B.) is grateful to the Deutsche Forschungsgemeinschaft (DFG) and the Gesellschaft für Schwerionenforschung Darmstadt (GSI) for financial support.

\section{References}

1. Mannervik, S., Physica Scripta 40, 28 (1989).

2. Blanke, J. H., Heckmann, P. H. and Träbert, E., Z. Phys. D3, 375 (1986).

3. Blanke, J. H., Fricke, B., Sepp, W.-D., Heckmann, P. H., Möller, G. and Wagner, C., Physica Scripta 42, 522 (1990).

4. Blanke, J. H., Fricke, B., Heinemann, D., Heckmann, P. H., Möller, G. and Träbert, E. (submitted to Physica Scripta).

5. Schneider, D., Bruch, R., Butscher, W. and Schwarz, W. H. E., Phys. Rev. A24, 1223 (1981).

6. Bruch, R., Chung, K. T., Luken, W. L. and Culberson, J. C., Phys. Rev. A31, 310 (1985).

7. Beck, D. R. and Nicolaides, C. A., Phys. Lett. 61A, 227 (1977).

8. Desclaux, J. P., Comp. Phys. Comm. 9, 31 (1975); private communication (1989).

9. Miecznik, G., Brage, T. and Froese Fischer, C., Physica Scripta 45, 436 (1992).

10. Blanke, J. H., Fricke, B., Heinemann, D., Sepp, W.-D., Thies, B., Göbenli, T., Heckmann, P. H., Möller, G. and Träbert, E., Z. Phys. D13, 1 (1989).

11. Blanke, J. H., Heckmann, P. H., Träbert, E. and Hucke, R., Physica Scripta 35, 780 (1987).

12. Wood, C. P. and Pyper, N. C., Mol. Phys. 41, 149 (1980).

13. Huang, K. N., Kim, Y. K., Cheng, K. T. and Desclaux, J. P., Phys. Rev. Lett. 48, 1245 (1982).

14. Träbert, E., Heckmann, P. H., Schlagheck, W. and Buttlar, H. v., Physica Scripta 21, 27 (1980).

15. Träbert, E., PhD thesis, Bochum (1978).

16. Kelly, R. L., "Atomic and Ionic Spectrum Lines Below 2000 \&, Hydrogen through Krypton", Oak Ridge National Laboratory Report, ORNL-5922, Washington, 1982.

17. White, H. E. and Eliason, A. Y., Phys. Rev. 44, 753 (1933).

18. Baudinet-Robinet, Y., Garnir, H. P. and Dumont, P. D., Phys. Rev. A28, 2169 (1983).

19. Lodwig, V. and Buttlar, H. v., Nucl. Instrum. Methods in Physics Research B31, 342 (1988). 